Figure 4 Same eye 4 months later. Late phase angiogram showing partial filling of the macroaneurysm (large arrow). Note blockage of background fluorescence due to residual old blood and retinal pigment epithelial window defect in the macular region.

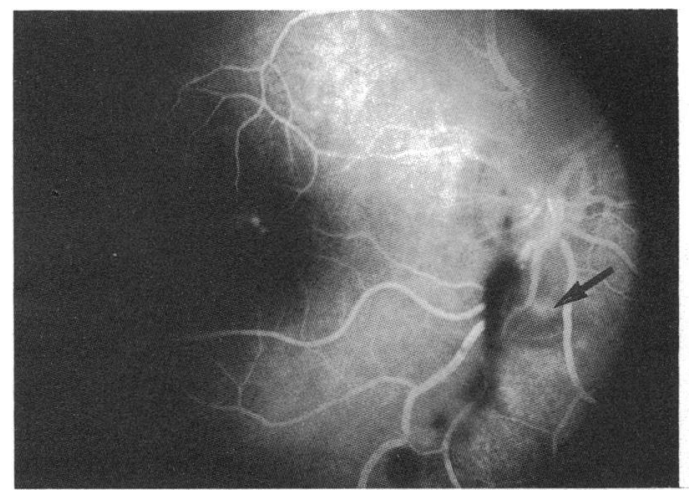

A follow up fluorescein angiograph revealed slow and incomplete filling of the aneurysmal dilatation, suggestive of partial obliteration of the lumen. An area of blocked background fluorescence was noted adjacent to and surrounding the aneurysm. Other areas of blocked choroidal fluorescence were seen, corresponding to the old haemorrhage. A retinal pigment epithelium window defect was noted in the macular region (Fig 4).

\section{Comment}

Since 1973, when Robertson ${ }^{1}$ first described retinal arteriolar macroaneurysms as a distinct clinical entity, to our knowledge this is the first report of an arteriolar macroaneurysm occurring in a young patient with congenital heart disease. ${ }^{1-8}$ The evolution of a macroaneurysm at the site of incomplete embolic occlusion of a branch retinal artery has been documented. ${ }^{3910} \mathrm{It}$ is postulated that embolic events result in focal vessel wall damage, predisposing to subsequent aneurysmal dilatation.

Cardiovascular evaluation of our patient revealed a congenital atrial septal defect; this may have caused retinal arterial embolic episodes, leading to focal arterial wall damage and development of a macroaneurysm. The focal area of periarterial sheathing seen distal to the macroaneurysm might also have been caused by embolic damage to the arterial wall. In addition, the focal area of thickening and loss of transparency of the arterial wall might represent involuted macroaneurysm, as has been postulated by Schatz et al. ${ }^{11}$

1 Robertson DM. Macroaneurysms of the retinal arteries. Trans Am Acad Ophthalmol Otolaryngol 1973; 77: 55-67.

2 Cleary PE, Kohner EM, Hamilton AM, Bird AC. Retinal macroaneurysms. Br F Ophthalmol 1975; 59: 355-61.

3 Lewis RA, Norton EWD, Gass JDM. Acquired arterial macroaneurysms of the retina. $B r \mathcal{F}$ Ophthalmol 1976; 60: 21-30.

Nadel A I, Gupta KK. Macroaneurysms of the retinal arteries. Arch Ophthalmol 1976; 94: $1092-6$

5 Palestine AG, Robertson DM, Goldstein BG. Macroaneurysms of the retinal arteries. Am f Ophthalmol 1982; 93: aneurys $164-71$.

6 Abdel-Khalek MN, Richardson J. Retinal macroaneurysm: natural history and guidelines of treatment. $\mathrm{Br} \mathcal{F}$ Ophthalmo 1986; 70: 2-11.

7 Lavin MJ, Marsh RJ, Peart S, Rehman A. Retinal arterial macroaneurysm: a retrospective study of 40 patients. BrF Ophthalmol 1987; 71: 284-91.

8 Rabb MF, Gagliano DA, Teske MP. Retinal arterial macroaneurysms. Surv Ophthalmol 1988; 33: 73-96.

9 Khalil M, Lorenzzetti MD. Acquired retinal macroaneurysms. Can $\mathcal{F}$ Ophthalmol $1979 ; 14$ : 163-8.

10 Wiznia RA. Development of a retinal artery macroaneurysm at the site of a previously detected retinal artery embolus. A $m$ f Ophthalmol 1992;114: 642-3.

11 Schatz H, Gitter K, Yannuzzi L, Irvine A. Retinal arterial macroaneurysms: a large collaborative study. Presented at the American Academy of Ophthalmology annual meeting, Chicago, November, 1980
Medical Eye Unit, St Thomas's Hospital, London

M B Eckstein

D J Spalton

Department of Clinical Neurophysiology, Brook General Hospital,

London

G Holder

Correspondence to:

D J Spalton, Medical Eye

Unit, St Thomas's Hospital,

London SE1 7EH.

Accepted for publication 18 May 1993

\title{
Visual loss from central serous retinopathy in systemic lupus erythematosus
}

\author{
M B Eckstein, D J Spalton, G Holder
}

Systemic lupus erythematosus (SLE) is a multisystem disease that affects about 1:2000 people in Britain, being nine times more common in women. The aetiology remains unknown but the disease is characterised by a wide range of circulating autoantibodies, particularly to nuclear antigens. Retinopathy may be due to a primary vasculitis that affects small and occasionally large retinal vessels or secondary to systemic complications such as hypertension and anaemia.' It is usually seen in patients who have active systemic disease and most commonly consists of a microvascular retinopathy characterised by cotton wool spots. ${ }^{2}$ Choroidopathy with serous detachments of the retina or pigment epithelium is much rarer and is usually seen in acutely ill or hypertensive patients. We report two SLE patients who presented with typical features of central serous retinopathy (CSR) and developed visual loss which failed to recover during 2 years of follow up.

\section{Case report}

CASE 1

A 44-year-old woman was diagnosed ackaving SLE in 1974 when she was 26 years old with positive anti-DNA and aptinuclear antibodies and a high anti-cardiotipin antibody titre. During the course of her illness she had developed neurological, renal, and cutaneous manifestations of the condition which included hypertension and epilepsy. 


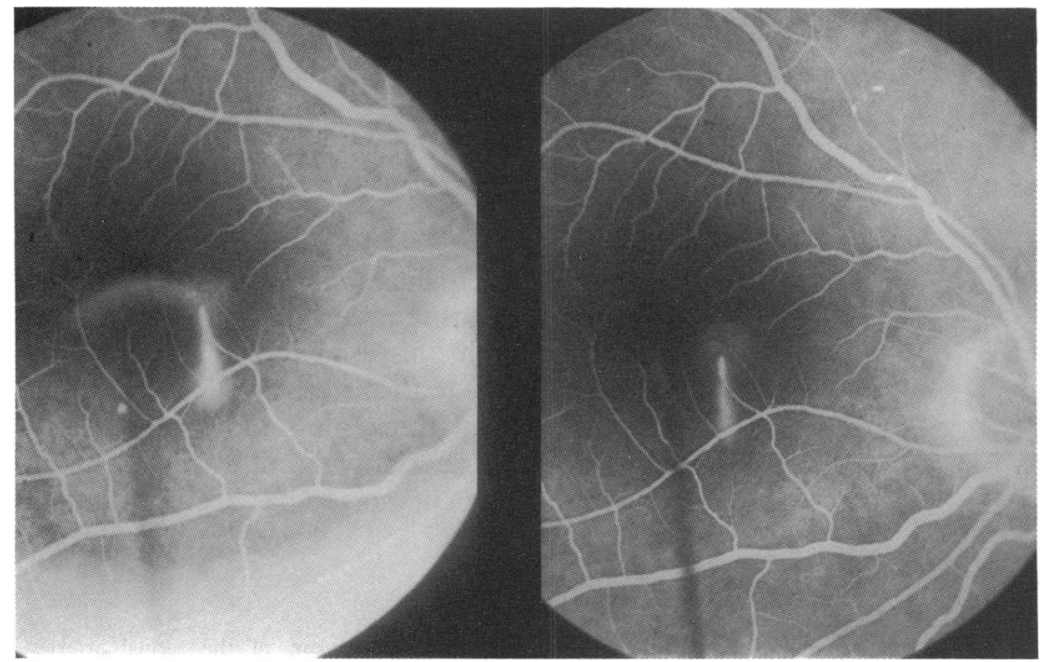

Figure 1 Fluorescein angiogram from case 1 showing the characteristic smoke stack appearance of central serous retinopathy. There is some pooling of fluorescein in the subretinal space in the later stages and a further area of leakage superotemporally is visible.

Figure 2 Pattern and flash $V E P$, pattern and flash $E R G$ from case 1 . The pattern ERG from the left eye shows a gross amplitude reduction of the P50 component as well as a delay in response.

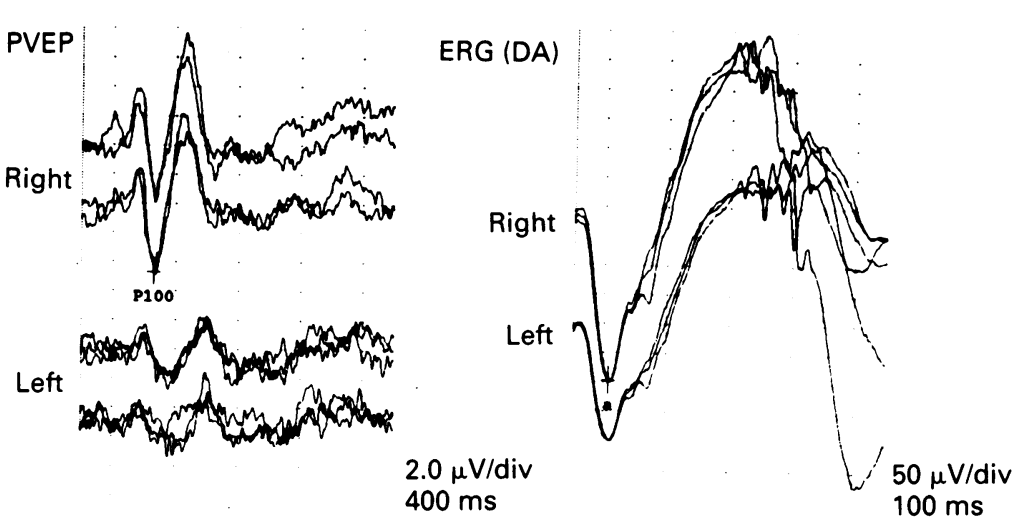

FVEP

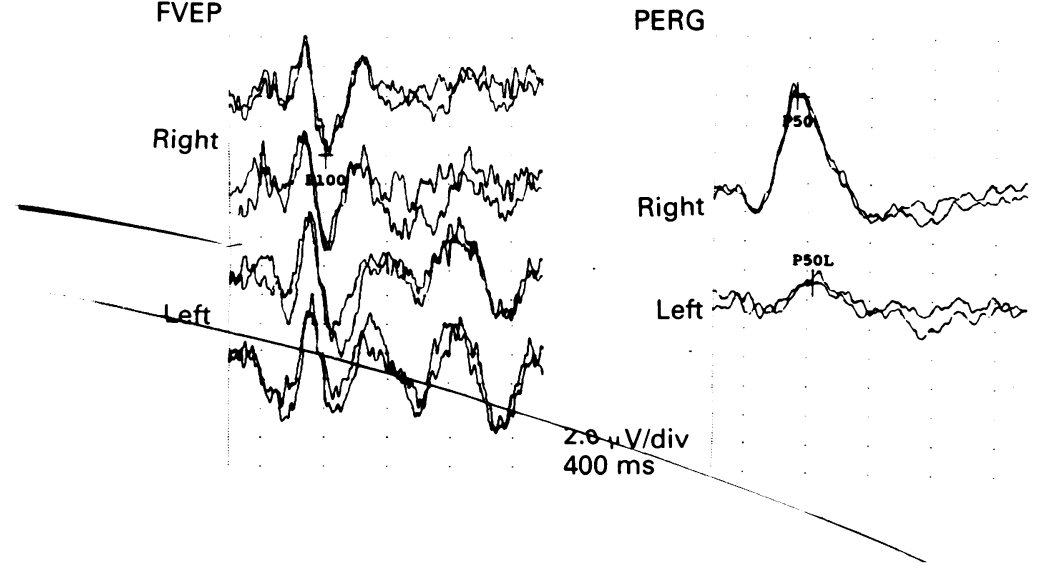

In March 1990 she coincidentally noticed that she was unable to see objects in the central field of her left eye. She had no history of any previous visual problem. The visual symptoms were painless and constant and her SLE was quiescent at this time with normal blood pressure. On examination her right eye was entirely normal. In her left eye, vision was $6 / 36, N 18$ with slightly depressed colour vision, a subtle retinal afferent pupillary defect (RAPD) and a central scotoma. There was a large central serous retinal detachment. The optic disc, peripheral retina, and vessels appeared normal. Fluorescein angiography confirmed the diagnosis of CSR, showing an area of fluorescein leakage through the retinal pigment epithelium (RPE) with dye accumulating under the sensory retina in the characteristic smoke stack appearance (Fig 1), choroidal filling was not obviously delayed or pathological. The CSR resolved without treatment over 4 months but vision failed to improve.

$1.0 \mu \mathrm{V} / \mathrm{div}$ $100 \mathrm{~ms}$
One year later the visual field and acuity were unchanged, the RAPD was more pronounced, the retina was flat, and the RPE appeared normal. Fluorescein angiography was unremarkable. Electrodiagnostic testing, comprising pattern and flash electroretinograms (ERGs) and visually evoked potentials (VEPs), was performed. Flash ERGs were normal in the affected eye, but the pattern ERG (PERG) showed a severe amplitude reduction in the P50 component accompanied by a latency increase. The pattern VEP was delayed, secondary to the PERG findings (Fig 2). Since February 1991 the visual signs have remained unchanged, in particular there is no optic nerve pallor or change in the RPE on funduscopy.

\section{CASE 2}

This 44-year-old woman presented at the age of 28 complaining of weight loss, hair loss, joint pains, and a skin rash. Antinuclear and antiDNA antibodies were present but there was no evidence of antiphospholipid antibodies. She was treated with prednisolone, azathioprine, and cyclosporin A. The disease remained relatively quiescent but in 1989 she developed symptoms of central nervous system involvement with limb weakness and coordination problems. A computed tomogram (CT) at that time was normal and her steroid dosage was increased.

In August 1990 she noticed a small well defined scotoma in the temporal field of her right eye. She was otherwise well and was normotensive. Corrected visual acuities were $6 / 6$ and N5 in both eyes with normal colour vision. There was no RAPD. Fundus examination revealed an area of serous retinal evaluation superotemporal to the disc. Fluorescein angiography demonstrated an area of fluorescein leakage in the early phase increasing as the angiogram progressed and compatible with a focal 'ink blot' leak of CSR. Another pigment epithelial lesion was present below the disc, seen more easily in the later part of the run; choroidal filling was not delayed (Fig 3).

A month later her vision had deteriorated to $6 / 36$ and the serous detachment was larger and involved the macula. The pigment epithelial lesion was treated with focal argon laser photocoagulation. Following this the retina flattened, and the fluorescein angiogram showed no leakage. Despite the resolution of the CSR her vision had deteriorated to $6 / 60$. She was admitted for a course of intravenous methylprednisolone but no visual improvement was obtained.

Electrodiagnostic testing was performed. The PERG was almost extinguished from the affected eye, but the flash ERG fell within the normal range. The pattern VEP was delayed and of reduced amplitude, secondary to the PERG findings (Fig 4). Two years later the fundus appearances and physical signs remained unchanged, in particular there was no optic atrophy.

\section{Comment}

These two patients presented with clinical signs of SLE which were confirmed by the appropriate 


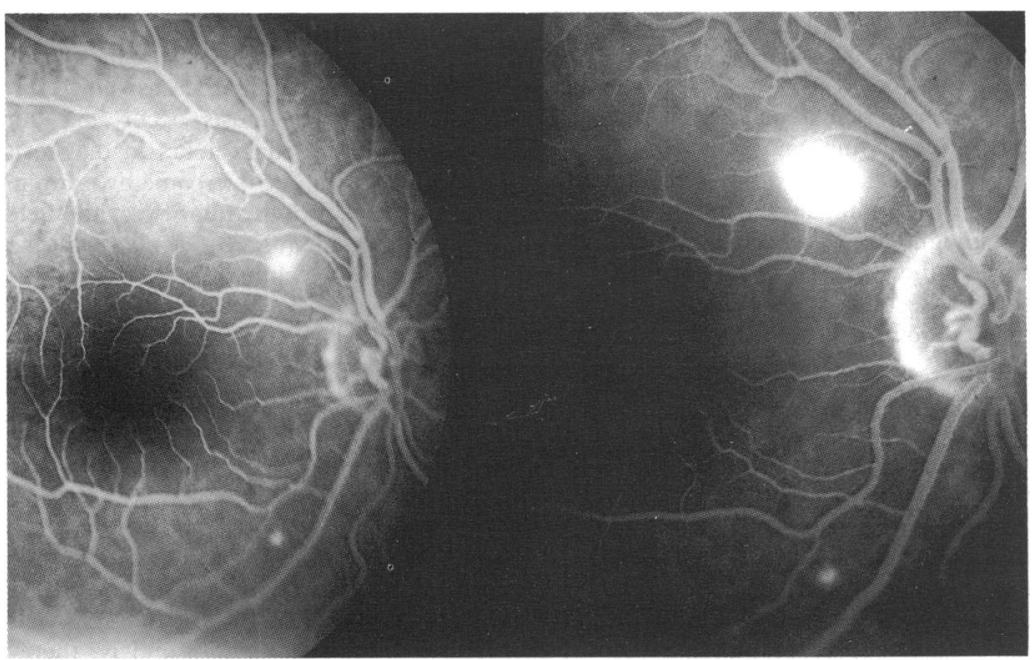

Figure 3 Fluorescein angiogram from case 2 showing the characteristic 'ink blot' leak of central serous retinopathy. Another smaller area of leakage is present inferotemporal to the optic disc. antibody tests. They developed typical angiographic appearances of CSR which resolved but unusually the visual loss did not recover. Choroidal and RPE involvement in SLE is rare. Jabs $e t a l^{3}$ described six patients with SLE and ocular involvement manifested by multifocal, serous elevations of the RPE and sensory retina. All of these patients had systemic vascular disease with active SLE. Four of the patients had macular involvement and visual loss but delayed choroidal perfusion was present in only one patient. Three of the patients had resolution of the serous retinal elevation with control of the systemic disease process, one patient progressed to a total exudative retinal detachment. Eight other patients with SLE and choroidopathy to our knowledge have been described. ${ }^{4-8}$ Five of these had serous macular detachments and three had more peripheral serous retinal detachments; all of these patients had evidence of systemic vascular disease.
Figure 4 Pattern and flash $V E P$, pattern and flash $E R G$ from case 2 . The $P 50$ component of the pattern $E R G$ from the right eye is virtually extinguished.

PVEP
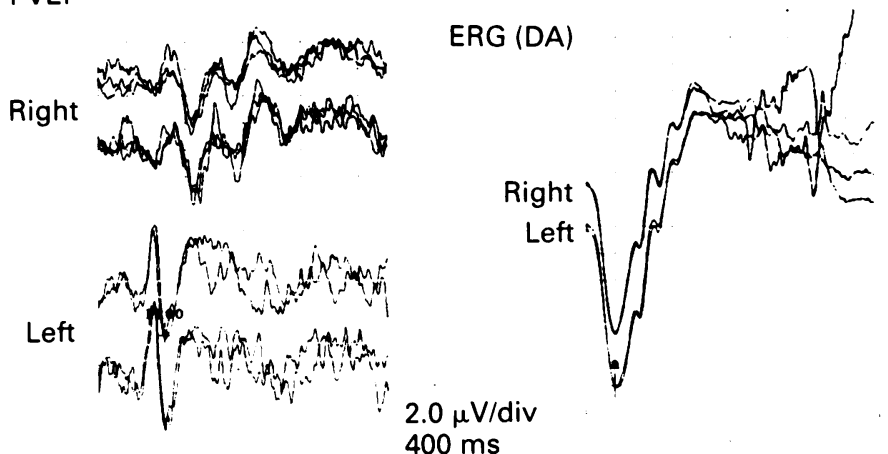

$50 \mu \mathrm{V} / \mathrm{div}$ $400 \mathrm{~ms}$ $100 \mathrm{~ms}$

FVEP

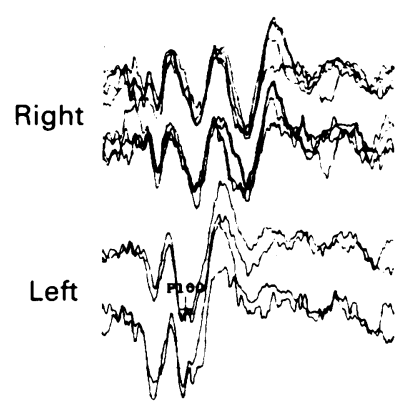

PERG

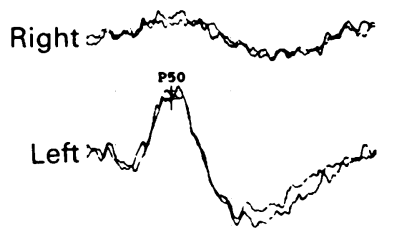

Central serous retinopathy normally has a good visual prognosis whether treated or untreated $^{9}$ and this is particularly so for the group of patients under 50 years of age, although in those who have the first episode after the age of 60 recovery of visual acuity is less certain. ${ }^{10}$ The reason for the permanent loss of vision in our two patients is uncertain; the possibilities are that this was due to retinal or optic nerve damage. The PERG contains two main components; a prominent positive component at approximately $52 \mathrm{~ms}$ (P50), and a large negative component (N95) at approximately $93 \mathrm{~ms}^{.1}$ It is our experience that P50 is rarely involved if the PERG is abnormal in optic nerve diseases; the abnormality is usually confined to N95. ${ }^{12}$ In contrast, P50 is invariably abnormal in macular disease, ${ }^{13}$ where latency increases can occasionally accompany an amplitude reduction. Latency increases in PERG P50 do not occur in optic nerve disease. The electrodiagnostic tests, in particular the PERG, therefore strongly suggest a retinal cause for the patients' continuing acuity reduction, the difference between the pattern and flash ERG further localising the pathology to the central retina. A potential mechanism for this may be decreased choroidal blood flow due to vasculitis of the choriocapillaris with breakdown of the outer blood-retinal barrier and subretinal leakage from choroidal vessels leading to secondary damage of the RPE and outer retina with loss of retinal function as a result of this. If this explanation is correct, however, it is surprising that we did not see any delay or abnormality in choroidal filling during fluorescein angiography in the acute phase, neither was there any evidence of RPE damage on long term follow up. An alternative explanation could be that following a breakdown of the outer blood-retinal barrier subretinal fluid accumulated in these two patients and this was toxic to the inner retina because of the underlying immune processes.

1 Spalton DJ. Systemic lupus erythematosus. In: Gold, DH, Weingeist TA, ed. The eye in systemic disease. Philadelphia Lippincott, 1990

2 Lanham JG, Barrie T, Kohner EM, Hughes GRV, SLE retinopathy: evaluation by fluorescein angiography. Ann Rheum Dis 1982; 41: 473-8.

3 Jabs D, Hanneken A, Schachat A, Fine S. Choriodopathy in systemic lupus erythematosus. Arch Ophthalmol 1988; 106 : $230-4$.

4 Matsuo T, Nakayama T, Koyama T, Matsuo N. Multifocal pigment epithelial damages with serous retinal detachment in systemic lupus erythematosus. Ophthalmologica 1987; in systemic

5 Diddie K, Aronson A, Ernest J. Chorioretinopathy in a case of systemic lupus erythematosus. Trans Am Ophthalmol Soc systemic lupus

6 Kinyoun J, Kalina R. Visual loss from choroidal ischaemia. Am F Ophthalmol 1986; 101: 650-6.

7 Coppeto J, Lessell S. Retinopathy in SLE. Arch Ophthalmo 1977; 95: 794-7.

8 Klinkhoff AV, Beattie CW, Chalmers A. Retinopathy in SLE: relationship to disease activity. Arthritis Rheum 1986; 29: 1152-6.

9 Mitchell Gilbert C, Owens S, Smith P, Fine S. Long term follow up of central serous chorioretinopathy. Brf Ophthalmol 1984; 68: 815-20.

10 Schatz H, Maderia D, Johnson R, McDonald R. Central serous chorioretinopathy occurring in patients 60 years of serous chorioretinopathy occurring in patie

11 Holder GE. Significance of abnormal pattern electroretinography in anterior visual pathway dysfunction. $\mathrm{Br} f$

12 Holder GE. The incidence of abnormal pattern electroretinography in optic nerve demyelination. Electroenceph Clin Neurophysiol 1991; 78: 18-26.

13 Holder GE. Pattern electroretinography in patients with delayed pattern visual evoked potentials due to distal anterior visual pathway dysfunction. $\mathcal{F}$ Neurol Neurosurg Psychiatry 1989; 52: 1364-8. 